\title{
Small cell carcinoma of the uterine cervix in pregnancy: A case report and review of the literature
}

\author{
QING WANG ${ }^{1}$, YI-HONG LIU ${ }^{1}$, LI XIE ${ }^{2}$, WEN-JING HU ${ }^{2}$ and BAO-RUI LIU ${ }^{2}$ \\ ${ }^{1}$ The Comprehensive Cancer Center of Nanjing Drum Tower Hospital, Clinical College of Nanjing Medical University; \\ ${ }^{2}$ Comprehensive Cancer Center of Drum Tower Hospital, Medical School of Nanjing University \\ and Clinical Cancer Institute of Nanjing University, Nanjing, Jiangsu 210008, P.R. China
}

Received February 22, 2014; Accepted September 26, 2014

DOI: $10.3892 / \mathrm{ol} .2014 .2668$

\begin{abstract}
The occurrence of cervical cancer during pregnancy is extremely rare, particularly small cell carcinoma. Small cell cervical carcinoma (SCCC) is a neuroendocrine tumor with a poor prognosis. This study presents the case of an 18-year-old female with stage IB2 SCCC complicated by pregnancy, who was treated with chemotherapy and radiotherapy. The patient was diagnosed shortly after giving birth, and is the youngest female case to be reported in the world. The patient was treated with cisplatin and etoposide chemotherapy and radiotherapy. Complete remission was achieved following neoadjuvant chemotherapy and radiotherapy, and the patient remains in clinical remission eight months following treatment. Cytological screening, colposcopy and if necessary, biopsy, and selective conization at 14-20 weeks should be considered in the patient evaluation.
\end{abstract}

\section{Introduction}

Small cell cervical carcinoma (SCCC) is a neuroendocrine tumor with great aggravation. It accounts for only $0.5-3 \%$ (1) of cases of cervical cancer and progresses rapidly with early lymphogenous and hemotagenous metastases. The incidence of cervical cancer during pregnancy is $\sim 1 / 2,000$ individuals. Patients with SCCC are more likely to exhibit lymph node metastases and lymph vascular space invasion. Although chemoradiation has been shown to improve survival in non-small cell carcinoma of cervix, the optimal initial therapeutic approach has not been identified in SCCC (2). Standard chemotherapy regimens, such as cisplatin and etoposide, are administered according to the management of small cell lung cancer. The five-year survival rate for SCCC ranges between 0 and 30\% (3). The present

Correspondence to: Dr Bao-Rui Liu, Comprehensive Cancer Center of Drum Tower Hospital, Medical School of Nanjing University and Clinical Cancer Institute of Nanjing University, 321 Zhongshan Road, Nanjing 210008, P.R. China

E-mail: baoruiliu@nju.edu.cn

Key words: cervix, neoadjustchemotherapy, pregnancy, radiotherapy, small cell carcinoma report describes a case of SCCC in an 18-year-old female, which occurred during pregnancy, and was treated with cisplatin and etoposide chemotherapy and then radiotherapy. Written informed consent was obtained from the patient.

\section{Case report}

An 18-year-old Chinese female presented to the Comphrensive Cancer Center of Drum Tower Hospital (Nanjing, China) with intermittent vaginal bleeding of $\sim 50 \mathrm{ml} /$ day, for 47 days following delivery. On June 3, 2013, a 6.0x6.0-cm exophytic friable mass was identified in the uterine cervix by colposcopic examination. On histological examination, the mass was diagnosed as SCCC (Fig 1). Immunohistochemistry revealed positive staining for chromogranin A ( $\mathrm{CgA})$, cytokeratin, cluster of differentiation (CD)56, synapsin (Syn), Ki67 (50\%+) and human papillomavirus (HPV). Magnetic resonance imaging (MRI) confirmed a $5.8 \times 5.7-\mathrm{cm}$ cervical carcinoma and swelling of the lymph nodes in the pelvis (Fig. 2). Based on the histological and radiographical observations (Fig. 1), the tumor was diagnosed as SCCC, and classified as International Federation of Gynecology and Obstetrics (FIGO) stage IB2 (4). Neoadjuvant chemotherapy (NACT) was administered, which consisted of four cycles of treatment with intravenous (i.v) cisplatin $\left(70 \mathrm{mg} / \mathrm{m}^{2}\right.$, days 1-3) and i.v etoposide (70 $\mathrm{mg} / \mathrm{m}^{2}$, days $1-5$ ) for $\sim 9$ weeks. Atypical vaginal spotting disappeared following the first cycle of therapy. After the fourth cycle of NACT, MRI revealed a $90 \%$ decrease in tumor size when compared with previous MRI scans of the cervical mass and an $80 \%$ decrease in size of the lymph nodes (Fig. 2). Three weeks after completing NACT, the patient received 3D intensity-modulated radiation therapy with a total dose of $44 \mathrm{~Gy}$ administered to the pelvis and 54 Gy to the pelvic lymph nodes. Image-guided high-dose-rate intracavity brachytherapy at a dose of $25 \mathrm{~Gy}$ in combination with low dose Nedaplat $\left(40 \mathrm{mg} / \mathrm{m}^{2}\right)$ was administered for eight weeks. MRI showed complete remission had been achieved following radiotherapy. On February 20, 2014, the patient was disease-free without signs of recurrence.

\section{Discussion}

The typical clinical manifestations of SCCC include irregular vaginal bleeding, exudation discharge and severe pain at 
advanced stages. The diagnosis of SCCC was aided by immunohistochemical staining for common neuroendocrine markers, including Syn, CgA, neuron-specific enolase (NSE) and CD56. Typically, SCCC samples exhibit 100, 96.0, 68.0, 76.0, 40.0, 84.0, 68.0 and $8.0 \%$ positivity for NSE, Syn, CD56, $\mathrm{CgA}$, thyroid transcription factor-1, epithelial membrane antigen, cytokeratin and S100 immunohistochemical biomarkers, respectively (5).

At the time of diagnosis, 60.0-82.0\% of SCCCs exhibit lymph-vascular space infiltration or pelvic lymph node metastasis (1). SCCC may also rapidly metastasize to the lungs, liver, brain, bones and pancreas. In a previous study, the rate of lymph node metastases in FIGO stage IB1 SCCC was $20 \%$, and in stages more advanced than IB1, the rate was $>50 \%$ (6). In patients who had relapsed following complete treatment, $67 \%$ exhibited hematogenous metastases and 34\% exhibited lymphogenous metastases, which indicated a higher probability of hematogenous metastases following first-line treatment. The overall five-year survival rates of 58 patients with SCCC were determined to be $\sim 55-85 \%$ for stages IA2-IB1, $25-30 \%$ for stage IB2-II and 0-16\% for stage III-IV (6). Liao et al (1) and Cohen et al (7) have reported that the prognosis of SCCC is associated with FIGO stage, tumor size, CgA levels, lymph node metastasis, depth of infiltration and treatment. Patients with FIGO stage I-IIA tumors, tumor size $\leq 4 \mathrm{~cm}$, negative CgA status, no lymph node metastasis and infiltrating depth of $<1 / 3$, exhibited a better prognosis. No significant differences in patient prognosis were observed with regard to NSE, Syn and lymphovascular space invasion. Cohen et al (7) found that in a multi-center study of 188 patients, the five-year survival rate for stage I-IIA patients who received a radical hysterectomy was $38.2 \%$, when compared with those who did not undergo surgery was $23.8 \%(\mathrm{P}<0.05)$. The correlation between HPV infection and the prognosis of SCCC is extremely controversial. One study reported that $\sim 76.2 \%$ (64/84) patients were HPV positive (8). HPV infection was associated with better disease-free survival, overall survival (OS) and local control. Vosmik et al (9) also demonstrated that patients with HPV positivity and high epidermal growth factor receptor expression had a high probability of achieving complete response ( $\mathrm{P}=0.038$ and $\mathrm{P}=0.044$, respectively).

Sood et al (10) compared 27 postpartum women, who were diagnosed within six months of giving birth, with 56 women diagnosed during pregnancy. A total of $48-49 \%$ of cervical cancers associated with pregnancy were found to be diagnosed within six months of delivery. Of the cervical cancers diagnosed during pregnancy, $89.3 \%$ cancers were stage I SCCC, $8.9 \%$ were stage II, $1.7 \%$ were stage $\geq \mathrm{III}$, whereas the rates were $70.4 \%, 26.0 \%$, and $3.7 \%$ in the women diagnosed after giving birth. Therefore, a higher proportion of early-stage tumors were diagnosed in the pregnant patients (11). Similar to the population of women who are not pregnant, the majority of cases of invasive cervical cancer were squamous cell carcinoma $(>80 \%)$. Of the remaining cases, the majority were adenocarcinomas, and SCCC was relatively rare.

To date, five cases of pregnant females with SCCC treated with NACT, followed by radical hysterectomy, have been reported. Gestation accelerates the progression of cervical carcinoma due to immunological suppression, high levels

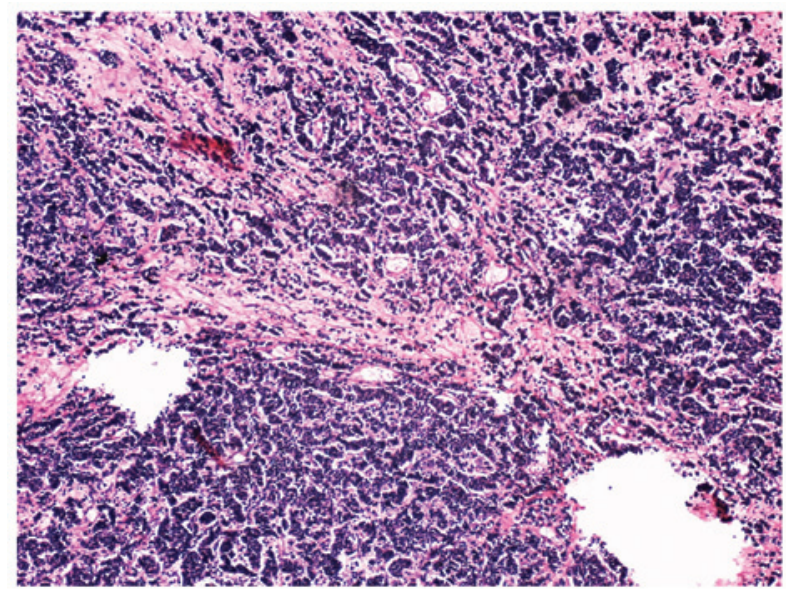

Figure 1. Hematoxylin and eosin-stained section revealing small cell cervical carcinoma with hyperchromatic nuclei and scant cytoplasm (magnification, x100).

of estrogen and an abundant cervical blood supply during pregnancy. In a study by Abeler et al (12), $40 \%$ patients were HPV-16-positive while $28 \%$ were HPV-18-positive (12). All cases of SCCC in pregnant females are shown in Table I. Pregnancy may augment the development of infections, in particular HPV infections. The five-year survival rate of cervical cancer during pregnancy is worse than cervical cancer alone, with rates of 70-78 and 87-92\%, respectively.

Small cell carcinoma is hypothesized to be sensitive to chemotherapy and radiotherapy. In the present study, first line systemic chemotherapy to eradicate the potential micrometastases was recommended due to the high probability of metastasis at early stages and the poor prognosis compared with other pathological types of cancer. Platinum-based chemotherapy in combination with pelvic and para-aortic nodal irradiation was administered. A multicenter, collaborative study reported that postoperative chemotherapy improved OS and progression-free survival (PFS) when compared with non-chemotherapy (6). The four-year PFS rate was 65\% in the group that received postoperative chemotherapy and $14 \%$ without postoperative chemotherapy, and the four-year OS rates were 65 and 29\%, respectively. Early stage patients who received post-chemotherapy with the etoposide regimen (cisplatin + etoposide), with or without radiation, exhibited an $83 \%$ three-year relapse-free survival, compared with $0 \%$ for those who did not receive adjuvant chemotherapy (13), indicating the importance of chemotherapy. No standard treatment regimens have been identified due to the low incidence of SCCC during pregnancy (2). As a result, standard regimens are selected according to the management of small cell lung cancer (3). The method for the treatment of SCCC includes radical surgery (RS) and/or radiotherapy, NACT combined with RS and/or radiotherapy or CCRT (2). Surgery is the first choice at early stages while system chemotherapy is critical at late stages. Katsumata et al (14) reported that NACT with BOMP (bleomycin, vincristine, mitomycin, and cisplatin) prior to RS did not improve the OS of patients with stage IB2, IIA and IIB cervical cancer. However, NACT reduced the proportion of patients who received postoperative RT. In a previous study, 10 patients were treated with a regimen of cisplatin, 


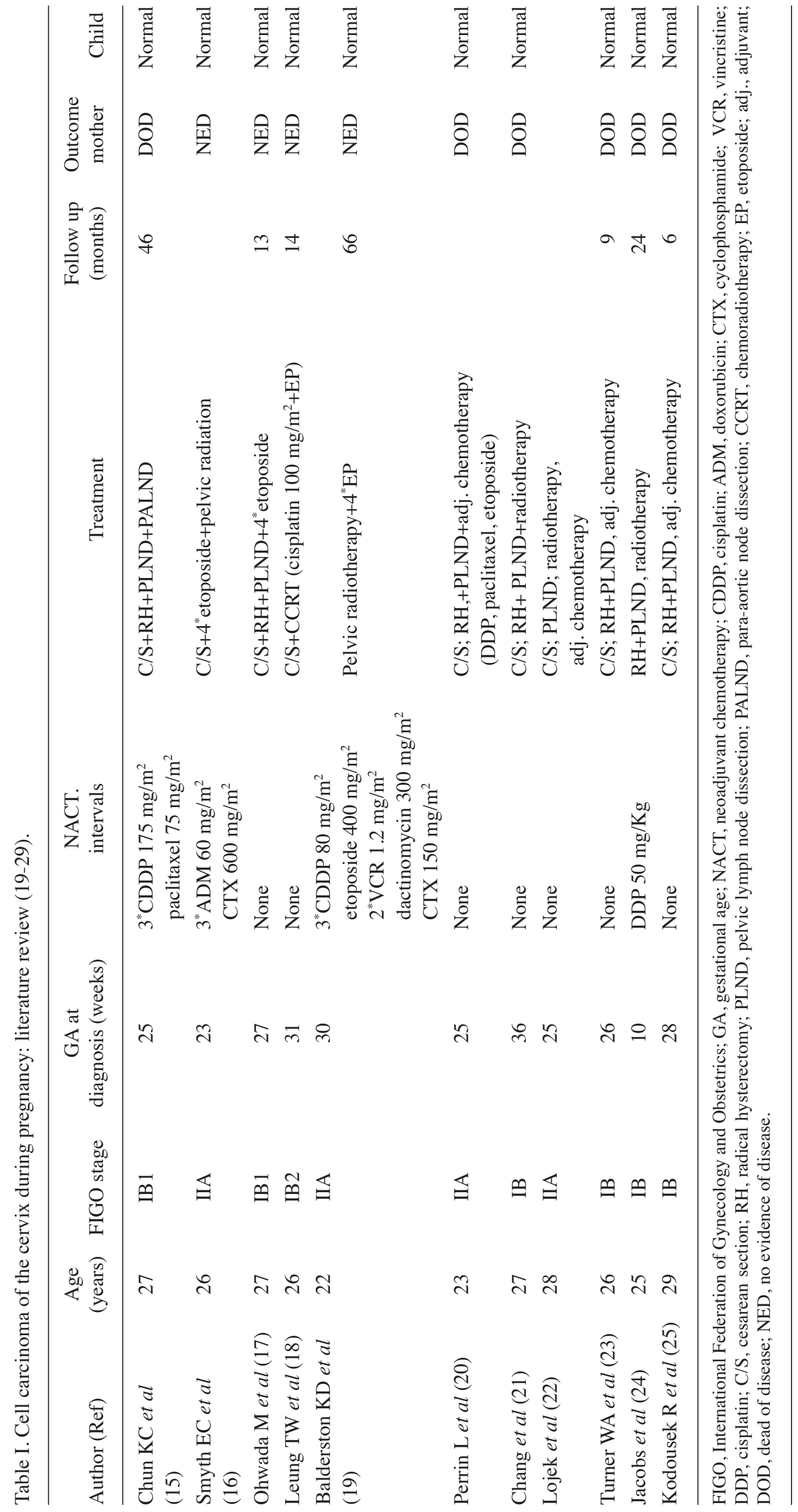



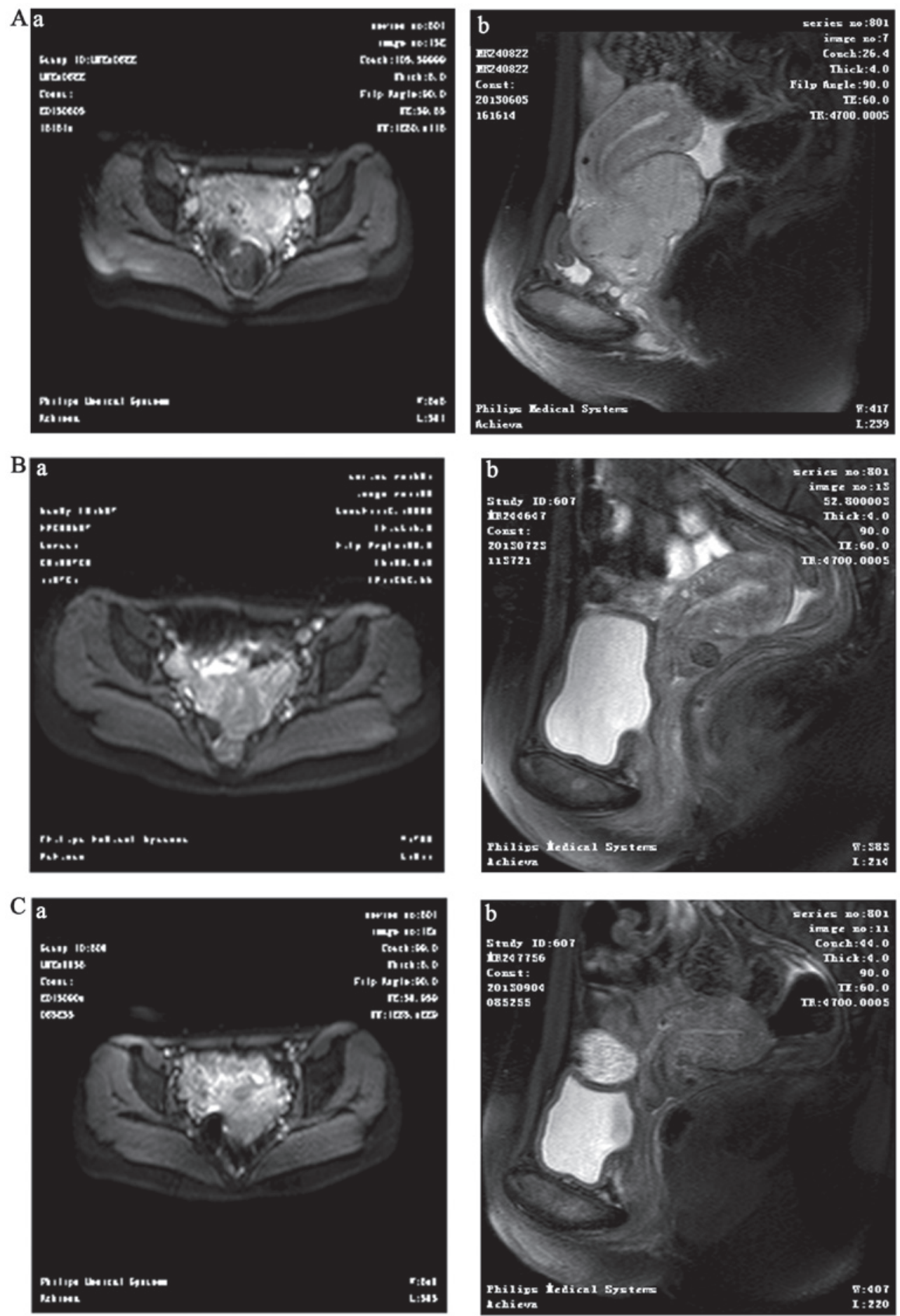

Figure 2. MRI of the pelvis. (A) Prior to treatment, MRI identified an $\sim 6.0 \times 6.0-\mathrm{cm}$ cervical carcinoma, and invasion of the posterior wall of vagina and pelvic lymph nodes. (B) Following two cycles of NACT for six weeks, a decrease in the volume of the vaginal mass was observed. (C) Following four cycles of NACT for 12 weeks, complete clinical remission was achieved. (a) transverse section and (b) median sagittal section. MRI, magnetic resonance imaging; NACT, neoadjuvant chemotherapy.

doxorubicin and etoposide followed by radical hysterectomy, pelvic lymphadenectomy and/or radical radiotherapy. The median duration of survival was 28 months (26). In another study, 14 patients with stage IA or IB SCCC received radical hysterectomy alone or in combination with pelvic radiotherapy. A total of $12 / 14$ patients succumbed to the disease within 31 months and the other two patients exhibited recurrence at 38 and 44 months (27). Although the sample sizes were small in these studies, the results indicated that chemotherapy followed by surgery may improve the outcome of patients. However, chemotherapy followed by radiotherapy is controversial (28-30), as a number of phase III clinical trials have demonstrated that chemotherapy followed by radiotherapy does not exhibit any significant survival benefit, however, a different toxicity profile appeared: alopecia $(87.9 \%$ vs. $4.1 \% ; \mathrm{P}<0.001)$ and neurotoxicity $(65.9 \%$ vs. $15.6 \% ; \mathrm{P}<0.001)$ were significantly higher in the patients with paclitaxel plus carboplatin followed by radiation. In the present study, the 
patient was diagnosed with stage IB2 SCCC, with an extensive mass in the cervix and lymph node metastasis in the pelvis. RS was not suitable and the patient underwent four cycles of NACT with cisplatin and etoposide. MRI of the pelvis showed complete remission of the mass in the cervix and lymph nodes. The patient subsequently received radical radiotherapy, due to being unsuitable for RS. A total of four additional cycles of cisplatin and etoposide were administered from February 2014, when the patient exhibited no evidence of relapse.

SCCC in pregnancy must be diagnosed as early as possible. Cytological screening, colposcopy and if required, biopsy, and selective conization at 14-20 weeks gestation should be considered in the patient evaluation (31). The stage of disease, gestational age at diagnosis and patient's preference must be considered for individualized treatment.

In conclusion, the present study reported a case of SCCC, which occurred during pregnancy. Treatment with NACT and radiotherapy resulted in complete clinical remission. Future clinical trials with a large cohorts are required to determine whether aggressive initial combined therapy, in particular NACT with radiotherapy, may improve the prognosis of advanced SCCC patients.

\section{References}

1. Liao LM, Zhang X, Ren YF, et al: Chromogranin A (CgA) as poor prognostic factor in patients with small cell carcinoma of the cervix: results of a retrospective study of 293 patients. PLoS One 7: e33674, 2012.

2. Delaloge S, Pautier P, Kerbrat P, et al: Neuroendocrine small cell carcinoma of the uterine cervix: what disease? What treatment? Report of ten cases and a review of the literature. Clin Oncol (R Coll Radiol) 12: 357-362, 2000.

3. Zivanovic O, Leitao MM Jr, Park KJ, et al: Small cell neuroendocrine carcinoma of the cervix: Analysis of outcome, recurrence pattern and the impact of platinum-based combination chemotherapy. Gynecol Oncol 112: 590-593, 2009.

4. FIGO Committee on Gynecologic Oncology: FIGO staging for carcinoma of the vulva, cervix, and corpus uteri. Int J Gynaecol Obstet 125: 97-98, 2014.

5. Li JD, Zhuang Y, Li YF, et al: A clinicopathological aspect of primary small-cell carcinoma of the uterine cervix: a single-centre study of 25 cases. Clin Pathol 64: 1102-1107, 2011.

6. Kuji S, Hirashima Y, Nakayama H, et al: Diagnosis, clinicopathologic features, treatment, and prognosis of small cell carcinoma of the uterine cervix: Kansai Clinical Oncology Group/Intergroup study in Japan. Gynecol Oncol 129: 522-527, 2013.

7. Cohen JG, Kapp DS, Shin JY, et al: Small cell carcinoma of the cervix: treatment and survival outcomes of 188 patients. Obstet Am J Obstet Gynecol 203: 347. e1-e6, 2010.

8. Lindel K, Burri P, Studer HU, et al: Human papillomavirus status in advanced cervical cancer: predictive and prognostic significance for curative radiation treatment. Int $\mathbf{J}$ Gynecol Cancer 15: 278-284, 2005.

9. Vosmik M, Laco J, Sirak I, et al: Prognostic Significance of human papillomavirus (HPV) status and expression of selected markers (HER 2/neu, EGFR, VEGF, CD34, p63, p53 and Ki67/MIB-1) on outcome after (chemo-) radiotherapy in patients with squamous cell carcinoma of uterine cervix. Pathol Oncol Res 20: 131-137, 2014.

10. Sood AK, Sorosky JI, Mayr N, et al: Cervical cancer diagnosed shortly after pregnancy: prognostic variables and Lishner M: Cancer in pregnancy. Annals of Oncology 14: 31-36, 2003.

11. Lishner M: Cancer in pregnancy. Ann Oncol 14 Suppl 3: iii31-iii36, 2003.
12. Abeler VM, Holm R, Nesland JM and Kjørstad KE: Small cell carcinoma of the cervix. A clinicopathologic study of 26 patients. Cancer 73: 672-677, 1994

13. Zivanovic O, Leitao MM Jr, Park KJ, et al: Small cell neuroendocrine carcinoma of the cervix: Analysis of outcome, recurrence pattern and the impact of platinum-based combination chemotherapy. Gynecol Oncol 112: 590-593, 2009.

14. Katsumata N, Yoshikawa H, Kobayashi H, et al: Phase III randomised controlled trial of neoadjuvant chemotherapy plus radical surgery vs radical surgery alone for stages IB2, IIA2, and IIB cervical cancer: a Japan Clinical Oncology Group trial (JCOG 0102). Br J Cancer 108: 1957-1963, 2013.

15. Chun KC, Kim DY, Kim JH, et al: Neoadjuvant chemotherapy with paclitaxel plus platinum followed by radical surgery in early cervical cancer during pregnancy: three case reports. Jpn J Clin Oncol 40: 694-698, 2010.

16. Smyth EC, Korpanty G, McCaffrey JA, et al: Small-cell carcinoma of the cervix at 23 weeks gestation. J Clin Oncol 28: e295-e297, 2010

17. Ohwada M, Suzuki M, Hironaka M, et al: Neuroendocrine small cell carcinoma of the uterine cervix showing polypoid growth and complicated by pregnancy. Gynecol Oncol 81: 117-119, 2001.

18. Leung TW, Lo SH, Wong SF. et al: Small cell carcinoma of the cervix complicated by pregnancy. Clin Oncol (R Coll Radiol) 11: 123-125, 1999.

19. Balderston KD, Tewari K, Gregory WT, et al: Neuroendocrine small cell uterine cervix cancer in pregnancy: long-term survival following combined therapy. Gynecol Oncol 71: 128-132, 1998.

20. Perrin L, Bell J and Ward B: Small cell carcinoma of the cervix of neuroendocrine rigin causing obstructed labor. Aust N Z J Obstet Gynaecol 36: 85-87, 1995.

21. Chang DH-C, Hsueh S and Soong YK: Small cell carcinoma of the uterine cervix with neurosecretory granules associated with pregnancy: a case report. J Reprod Med 39: 537-540, 1994

22. Lojek MA, Fer MF, Kasselberg AG, et al: Cushing's syndrome with small cell carcinoma of the uterine cervix. Am J Med 69: 140-144, 1980

23. Turner WA, Gallup DG, Talledo OE, et al: Nueroendocrine carcinoma of the uterine cervix complicated by pregnancy: case report and review of the literature. Obstet Gynecol 67 (3 Suppl): 80S-83S, 1986.

24. Jacobs AJ, Marchevsky A, Gordon RE, et al: Oat cell carcinoma of the uterine cervix in a pregnant woman treated with cisdiamminedichloroplatinum: case report. Gynecol Oncol 9: 405-410, 1980.

25. Kodousek R, Gazarek F, Dusek J and Dostál S: Malignant apudoma (argyrophil cell cancer) of the uterine cervix in a 24-year-old woman in pregnancy. Cesk Pathol 12: 37-44, 1976 (In Czech).

26. Morris M, Gershenson DM, Eifel P, et al: Treatment of small cellcarcinoma of the cervix with cisplatin. doxorubicin and etoposide. Gynecol Oncol 47: 62-65, 1992.

27. Sheets EE, Berman ML, Hrountas CK. et al: Surgically treated, early stage small cell cervical carcinoma. Obstet Gynecol 71: 10-14, 1988.

28. Sehouli J, Runnebaum IB, Fotopoulou C, et al: A randomized phase III adjuvant study in high-risk cervical cancer: simultaneous radiochemotherapy with cisplatin (S-RC) versus systemic paclitaxel and carboplatin followed by percutaneous radiation (PC-R): a NOGGO-AGO Intergroup Study. Ann Oncol 23: 2259-2264, 2012.

29. Herod J, Burton A, Buxton J, et al: A randomised, prospective, phase III clinical trial of primary bleomycin, ifosfamide and cisplatin (BIP) chemotherapy followed by radiotherapy versus radiotherapy alone in inoperable cancer of the cervix. Ann Oncol, 11: 1175-1181, 2000.

30. Symonds RP, Habeshaw T, Reed NS, et al: The Scottish and Manchester randomised trial of neo-adjuvant chemotherapy for advanced cervical cancer. Eur J Cancer 36: 994-1001, 2000.

31. Nguyen C, Montz FJ and Bristow RE: Management of stage I cervical cancer in pregnancy. Obstet Gynecol Surv 55: 633-643, 2000 